\title{
Specifications for Managed Strings, Second Edition
}

Hal Burch (Software Engineering Institute) Fred Long (University of Wales, Aberystwyth) Raunak Rungta (Software Engineering Institute) Robert Seacord (Software Engineering Institute)

David Svoboda (Software Engineering Institute)

May 2010

TECHNICAL REPORT

CMU/SEI-2010-TR-018

ESC-TR-2010-018

CERT ${ }^{\circledR}$ Program

Unlimited distribution subject to the copyright.

http://www.cert.org 
This report was prepared for the

SEI Administrative Agent

$\mathrm{ESC} / \mathrm{XPK}$

5 Eglin Street

Hanscom AFB, MA 01731-2100

The ideas and findings in this report should not be construed as an official DoD position. It is published in the interest of scientific and technical information exchange.

This work is sponsored by the U.S. Department of Defense. The Software Engineering Institute is a federally funded research and development center sponsored by the U.S. Department of Defense.

Copyright 2010 Carnegie Mellon University.

\section{NO WARRANTY}

THIS CARNEGIE MELLON UNIVERSITY AND SOFTWARE ENGINEERING INSTITUTE MATERIAL IS FURNISHED ON AN "AS-IS" BASIS. CARNEGIE MELLON UNIVERSITY MAKES NO WARRANTIES OF ANY KIND, EITHER EXPRESSED OR IMPLIED, AS TO ANY MATTER INCLUDING, BUT NOT LIMITED TO, WARRANTY OF FITNESS FOR PURPOSE OR MERCHANTABILITY, EXCLUSIVITY, OR RESULTS OBTAINED FROM USE OF THE MATERIAL. CARNEGIE MELLON UNIVERSITY DOES NOT MAKE ANY WARRANTY OF ANY KIND WITH RESPECT TO FREEDOM FROM PATENT, TRADEMARK, OR COPYRIGHT INFRINGEMENT.

Use of any trademarks in this report is not intended in any way to infringe on the rights of the trademark holder.

Internal use. Permission to reproduce this document and to prepare derivative works from this document for internal use is granted, provided the copyright and "No Warranty" statements are included with all reproductions and derivative works.

External use. This document may be reproduced in its entirety, without modification, and freely distributed in written or electronic form without requesting formal permission. Permission is required for any other external and/or commercial use. Requests for permission should be directed to the Software Engineering Institute at permission@sei.cmu.edu.

This work was created in the performance of Federal Government Contract Number FA8721-05-C-0003 with Carnegie Mellon University for the operation of the Software Engineering Institute, a federally funded research and development center. The Government of the United States has a royalty-free government-purpose license to use, duplicate, or disclose the work, in whole or in part and in any manner, and to have or permit others to do so, for government purposes pursuant to the copyright license under the clause at 252.227-7013. 


\section{Table of Contents}

Acknowledgments $\quad$ iii

$\begin{array}{ll}\text { Abstract } & \text { v }\end{array}$

1 Introduction $\quad 1$

$\begin{array}{lll}1.1 & \text { String Manipulation Errors } & 1\end{array}$

1.2 Proposed Solution 1

1.3 The Managed String Library 2

1.4 Wide Character and Null-Terminated Byte Strings 2

2 Library $\quad \mathbf{5}$

2.1 Use of errno $\quad 5$

2.2 Runtime-Constraint Violations $\quad 5$

$\begin{array}{lll}2.3 & \text { Errors }<\text { errno.h> } & 5\end{array}$

2.4 Common Definitions <stddef.h> 6

2.5 Integer Types <stdint.h> 6

2.6 Managed String Type <string_m.h> $\quad 6$

$\begin{array}{lll}2.7 & \text { General Utilities <stdlib.h> } & 7\end{array}$

$3 \quad$ Library Functions $\quad 9$

$\begin{array}{ll}3.1 & \text { Utility Functions }\end{array}$

3.1.1 The isnull m Function $\quad 9$

3.1.2 The isempty m Function 9

3.1.3 Creating a Managed String 9

3.1.4 The isntbs_m Function 12

3.1.5 The iswide $m$ Function 12

3.1.6 The strdelete_m Function 13

3.1.7 The strlen_m Function 13

3.1.8 Extracting a Conventional String 13

3.1.9 The strdup m Function 14

3.2 Copying Functions $\quad 15$

3.2.1 Unbounded String Copy 15

3.2.2 The strncpy m Function 16

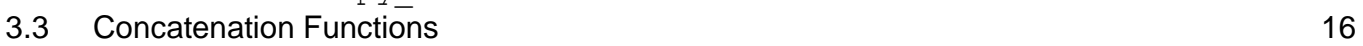

3.3.1 Unbounded Concatenation 16

3.3.2 Bounded Concatenation $\quad 17$

$\begin{array}{ll}3.4 & \text { Comparison Functions } \\ \end{array}$

$\begin{array}{ll}3.4 .1 & 19\end{array}$

$\begin{array}{ll}3.4 .2 & \text { Bounded String Comparison }\end{array}$

\begin{tabular}{ll}
3.5 & Search Functions \\
\hline
\end{tabular}

3.5.1 The strtok m Function $\quad 22$

3.5.2 The cstrch $\bar{r}$ m Function $\quad 22$

3.5.3 The wstrchr m Function 23

3.5.4 The strspn_m Function 23

3.5.5 The cstrspn m Function $\quad 23$

3.5.6 The wstrspn_m Function $\quad 24$

3.5.7 The strcspn_m Function $\quad 24$

3.5.8 The cstrcspn_m Function $\quad 25$

3.5.9 The wstrcspn_m Function $\quad 25$

3.6 Configuration Functions 26 
3.6.1 The setcharset_m Function $\quad 26$

3.6.2 The setmaxlen_m Function $\quad 26$

$\begin{array}{lll}3.7 & \text { Functions Derived from printf } & 26\end{array}$

$\begin{array}{ll}\text { 3.7.1 The sprintf_m Function } & 27\end{array}$

3.7.2 The vsprintf_m Function $\quad 27$

$\begin{array}{ll}\text { 3.7.3 The printf_m Function } & 28\end{array}$

3.7.4 The vprintf_m Function $\quad 28$

3.7.5 The fprintf_m Function $\quad 29$

3.7.6 The vfprintf_m Function $\quad 29$

$\begin{array}{lll}3.8 & \text { Functions Derived from scanf } & 30\end{array}$

3.8.1 The sscanf_m Function $\quad 30$

3.8.2 The vsscanf_m Function $\quad 30$

3.8.3 The scanf_m Function $\quad 31$

3.8.4 The vscanf $m$ Function 31

3.8.5 The fscanf $m$ Function $\quad 32$

3.8.6 The vfscanf m Function 32

3.9 String Slices $\quad 33$

3.9.1 The strslice m Function 33

3.9.2 The strleft_m Function 33

3.9.3 The strright_m Function $\quad 34$

3.9.4 The cchar_m Function $\quad 34$

3.9.5 The wchar_m Function $\quad 35$

$\begin{array}{lr}\text { References } & 37\end{array}$ 


\section{Acknowledgments}

The authors want to thank David Keaton and Martin Sebor for their valuable contributions to this technical report. 
iv | CMU/SEI-2010-TR-018 


\section{Abstract}

This report describes a managed string library for the $\mathrm{C}$ programming language. Many software vulnerabilities in $\mathrm{C}$ programs result from the misuse of manipulation functions for standard $\mathrm{C}$ strings. Programming errors common to string-manipulation logic include buffer overflow, truncation errors, string termination errors, and improper data sanitization. The managed string library provides mechanisms to eliminate or mitigate these problems and improve system security. The CERT $^{\circledR}$ Program, which is part of the Carnegie Mellon ${ }^{\circledR}$ Software Engineering Institute, provides a proof-of-concept implementation of the managed string library on its Secure Coding web pages. 
vi | CMU/SEI-2010-TR-018 


\section{Introduction}

\subsection{String Manipulation Errors}

Many software vulnerabilities in $\mathrm{C}$ programs arise through the misuse of manipulation functions for standard C strings. String manipulation programming errors include truncation errors, termination errors, improper data sanitization, and buffer overflow through string copying.

Buffer overflow can easily occur during string copying if the fixed-length destination of the copy is not large enough to accommodate the source string. This is a particular problem when the source is user input, which is potentially unbounded. The usual programming practice is to allocate a character array that is generally large enough. However, this fixed-length array can still be exploited by a malicious user who supplies a carefully crafted string that overflows the array in a way that compromises the security of the system. This is the most common exploit in fielded C code today.

In attempting to overcome the buffer overflow problem, some programmers limit the number of characters that are copied. This can result in strings being improperly truncated, which in turn results in a loss of data that can lead to a different type of software vulnerability.

A special case of truncation error is a termination error. Many of the standard C string functions rely on strings being null-terminated. However, the length of a string does not include the null character. If just the non-null characters of a string are copied, the resulting string may not be properly terminated. A subsequent access may run off the end of the string, corrupting data that should not have been touched.

Finally, inadequate data sanitization can also lead to software vulnerabilities. To function properly, many applications require that data does not contain certain characters. Ensuring that the strings used by the application do not include illegal characters can often prevent malicious users from exploiting an application.

\subsection{Proposed Solution}

A secure string library should provide facilities to guard against the programming errors described above. Furthermore, it should satisfy the following requirements:

- Operations should succeed or fail unequivocally.

- The facilities should be familiar to $\mathrm{C}$ programmers to facilitate both their adoption and the conversion of existing code.

- Using the facilities should not involve any surprises. The new facilities should have semantics similar to the manipulation functions for standard $\mathrm{C}$ strings. Again, this will help with the conversion of legacy code.

Of course, some compromises are needed to meet these requirements. For example, it is not possible to completely preserve the existing semantics and provide protection against the programming errors described above. 
Libraries that provide string manipulation functions can be categorized as static or dynamic. Static libraries rely on fixed-length arrays. A static approach cannot overcome the errors described above as easily as a dynamic approach. With a dynamic approach, strings are resized as necessary, but a consequence is that memory can be exhausted if input is not limited. To mitigate this problem, the managed string library allows for the specification of a per-string maximum length.

\subsection{The Managed String Library}

The CERT ${ }^{\circledR}$ Program, which is part of the Carnegie Mellon ${ }^{\circledR}$ Software Engineering Institute, has developed a proof-of-concept implementation of the managed string library in response to the need for a string library that could improve the quality and security of newly developed C language programs while eliminating obstacles to widespread adoption and possible standardization [CERT 2009]. The managed string library is available on the CERT Secure Coding website, http://www.cert.org/secure-coding/managedstring.html.

The managed string library is based on a dynamic approach where memory is allocated and reallocated as required. This approach eliminates the possibility of unbounded copies, nulltermination errors, and truncation by ensuring adequate space is always available for the resulting string (including the terminating null character).

A runtime-constraint violation occurs when memory cannot be allocated. In this way, the managed string library accomplishes the goal of succeeding or failing unequivocally.

The managed string library also provides a mechanism for dealing with data sanitization by (optionally) checking that all characters in a string belong to a predefined set of safe characters.

\subsection{Wide Character and Null-Terminated Byte Strings}

A number of managed string functions

- $\quad$ accept either a null-terminated byte string or a wide character string as input

- $\quad$ provide one of those string types as a return value

The managed string library works equally well with either type of string. For example, it is possible to create a managed string from a wide character string and then extract a null-terminated byte string (or vice versa). It is also possible to copy a null-terminated byte string and then concatenate a wide character string. Managed string functions will handle conversions implicitly when possible. If a conversion cannot be performed, the operation is halted and a runtime-constraint error is reported.

Strings are maintained in the format in which they are initially provided, until such a time that a conversion is necessary. String promotions are relatively simple: performing an operation on two null-terminated byte strings results in a null-terminated byte string, an operation on a nullterminated byte string and a wide character string results in a wide character string, and operations on two wide character strings result in a wide character string. Conversions are performed as necessary in the locale defined at the time the conversion occurs.

\footnotetext{
${ }^{\circledR}$ Carnegie Mellon and CERT are registered in the U.S. Patent and Trademark Office by Carnegie Mellon University.
} 
Managed strings also support the definition of a restricted character set that identifies the set of allowable characters for the string. When an operation requires that a null-terminated byte string be converted to a wide character string, the restricted character set is also converted as part of the operation. 
4 | CMU/SEI-2010-TR-018 


\section{Library}

\subsection{Use of errno}

An implementation may set errno for the functions defined in this technical report but is not required to do so.

\subsection{Runtime-Constraint Violations}

Most functions in this technical report include as part of their specifications a list of runtimeconstraints, which are requirements on the program using the library. Despite its name, a runtime-constraint is not a kind of constraint. Implementations shall verify that the runtime-constraint for a library function are not violated by the program.

Implementations shall check that the runtime-constraints specified for a function are met by the program. If a runtime-constraint is violated, the implementation shall call the currently registered constraint handler (see set_constraint_handler in Section 2.7). Multiple runtimeconstraint violations in the same call to a library function result in only one call to the constraint handler. It is unspecified which one of the multiple runtime-constraint violations cause the handler to be called.

Sometimes the runtime-constraints section for a function states an action to be performed if a runtime-constraint violation occurs. Such actions are performed before calling the runtime-constraint handler. Sometimes the runtime-constraints section lists actions that are prohibited if a runtimeconstraint violation occurs. Such actions are prohibited to the function both before the handler is called and after the handler returns.

The runtime-constraint handler may not return. If it does, the library function whose runtimeconstraint was violated shall return some indication of failure as given by the returns section in the function's specification.

Although runtime-constraints replace many cases of undefined behavior from ISO/IEC 9899:1999 [ISO/IEC 1999], undefined behavior can still occur. Implementations are free to detect any case of undefined behavior and treat it as a runtime-constraint violation by calling the runtimeconstraint handler. This license comes directly from the definition of undefined behavior.

\subsection{Errors $<$ errno.h>}

The header <errno. $\mathrm{h}>$ defines the following type, which is int:

$$
\text { errno_t }
$$




\subsection{Common Definitions $<$ stddef. $h>$}

The $<$ stddef. $h>$ header defines the following type, which is si ze_t: ${ }^{1}$

$$
\text { rsize_t }
$$

\subsection{Integer Types <stdint.h>}

The <stdint. $\mathrm{h}>$ header defines the following macro, which expands to a value of type size_t. ${ }^{2}$

$$
\text { RSIZE_MAX }
$$

Functions that have parameters of type rsize_t consider it a runtime-constraint violation if the values of those parameters are greater than RSIZE_MAX.

\section{Recommended Practice}

Extremely large object sizes are frequently a sign that an object's size was calculated incorrectly. For example, negative numbers appear as very large positive numbers when converted to an unsigned type such as size_t. Also, some implementations do not support objects as large as the maximum value that can be represented by type size_t.

For those reasons, it is sometimes beneficial to restrict the range of object sizes to detect programming errors. For implementations targeting machines with large address spaces, RSIZE_MAX should be defined as the smaller of the size of the largest object supported or ( SIZE_MAX $>>1$ ), even if this limit is smaller than the size of some legitimate, but very large, objects. Implementations targeting machines with small address spaces may wish to define RSIZE_MAX as SIZE_MAX, which means that no object size is considered a runtime-constraint violation.

\subsection{Managed String Type $<$ string_m.h $>$}

The $<$ string_m. $h>$ header defines an abstract data type:

$$
\text { typedef struct string_mx string_mx; }
$$

The structure referenced by this data type is private and implementation defined. All managed strings of this type have a maximum string length that is determined when the string is created. For functions that have parameters of type pointer to string_mx, it is a runtime-constraint violation if the maximum length of a managed string is exceeded.

Managed strings can also have a defined set of valid characters that can be used in the string. For functions that have parameters of type pointer to string_mx, it is a runtime-constraint violation if a managed string contains invalid characters. For functions that have parameters of type pointer

\footnotetext{
1 See the description of the RSIZE_MAX macro in $<$ stdint. $h\rangle$.

2 The RSIZE_MAX macro does not have to expand to a constant expression.
} 
to string_mx, it is a runtime-constraint violation if the request requires allocating more memory than is available. ${ }^{3}$

Managed strings support both null and empty strings. An empty string is one that has zero characters. A null string is an uninitialized string or a string that has been explicitly set to null.

For computing the length of a string to determine if the maximum length is exceeded, the length of a null-terminated byte string is the number of bytes, and the length of a wide character string is the number of characters. Thus, promoting a multi-byte, null-terminated byte string may change its length. Constants strings can be created by defining the structure string_mx to be constant and then calling the const_strcreate_m function.

\subsection{General Utilities <stalib.h>}

The header <stdlib.h> defines six types:

- errno_t, which is type int

- rsize_t, which is type size_t

- constraint_handler_t, which has the definition

typedef void (*constraint_handler_t) (

const char * restrict msg,

void * restrict ptr,

errno_t error);

- malloc_handler_t, which has the definition

typedef void * (*malloc_handler_t) (

size_t size);

- realloc_handler_t, which has the definition

typedef void * (*realloc_handler_t) (

void * ptr, size_t size);

- free_handler_t, which has the definition

typedef void (*free_handler_t) (void *ptr);

3 The library depends on malloc () and $r e a l l o c($ ) returning a null pointer to signify insufficient memory. On some systems, particularly systems using optimistic memory allocation schemes, mal loc () may return a nonnull pointer even when there is insufficient memory. On systems where there is no such mechanism to detect out-of-memory conditions, the library will not be able to properly validate this condition. 
8 | CMU/SEI-2010-TR-018 


\section{Library Functions}

\subsection{Utility Functions}

\subsubsection{The isnull_m Function}

\section{Synopsis}

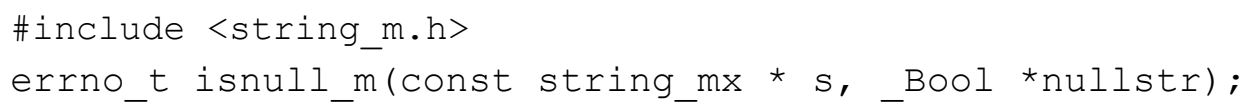

\section{Runtime-Constraints}

s shall reference a valid managed string. nullstr shall not be a null pointer.

\section{Description}

The isnull_m function tests whether the managed string $s$ is null and delivers this result in the parameter referenced by nullstr, given the managed string s.

\section{Returns}

The isnull_m function returns 0 if no runtime-constraints were violated. Otherwise, a nonzero value is returned.

\subsubsection{The isempty_m Function}

\section{Synopsis}

\#include <string_m.h>

errno_t isempty_m(const string_mx * s, Bool *emptystr);

\section{Runtime-Constraints}

s shall reference a valid managed string. emptystr shall not be a null pointer.

\section{Description}

The isempty_m function tests whether the managed string $s$ is empty and delivers this result in the parameter referenced by emptystr, given the managed string $s$.

\section{Returns}

The isempty_m function returns 0 if no runtime-constraints were violated. Otherwise, a nonzero value is returned.

\subsubsection{Creating a Managed String}

\subsubsection{The strcreate_m Function}

\section{Synopsis}

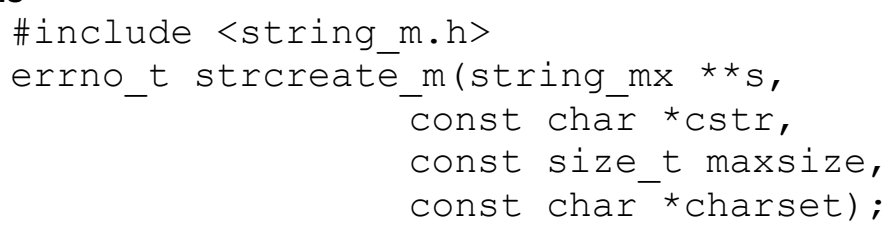




\section{Runtime-Constraints}

$\mathrm{s}$ shall not be a null pointer. charset shall not be an empty string (denoted by " "). Invalid characters are not present in the $\mathrm{C}$ string passed to the function.

\section{Description}

The strcreate_m function creates a managed string, referenced by $s$, given a conventional string cstr (which may be null or empty). maxsize specifies the maximum length of the string in characters. If maxsize is 0 , the system-defined maximum size is used. charset restricts the set of allowable characters to those in the null-terminated byte string cstr (which may be empty). If charset is a null pointer, no restricted character set is defined. If specified, duplicated characters in a charset are ignored. Characters in the charset may be provided in any order. The $\backslash 0$ character cannot be specified as part of charset.

\section{Returns}

The strcreate_m function returns 0 if no runtime-constraints were violated. Otherwise, a nonzero value is returned.

\subsubsection{The wstrcreate_m Function}

\section{Synopsis}

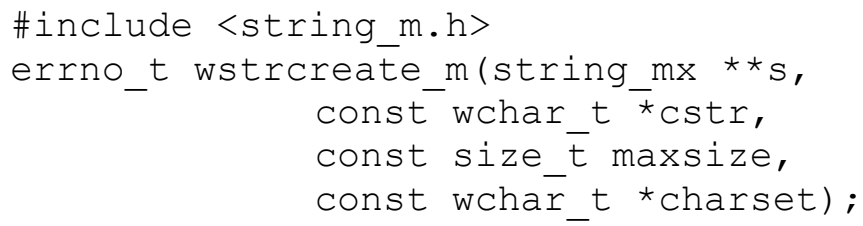

\section{Runtime-Constraints}

$\mathrm{S}$ shall not be a null pointer. charset shall not be an empty string (denoted by L""). Invalid characters are not present in the $\mathrm{C}$ string passed to the function.

\section{Description}

The wstrcreate_m function creates a managed string, referenced by $s$, given a wide character string cstr (which may be null or empty). maxsize specifies the maximum size of the string in characters. If maxsize is zero, the system-defined maximum length is used. charset restricts the set of allowable characters to those in the wide character string cstr (which may be empty). If charset is a null pointer, no restricted character set is defined. Characters in the charset may be provided in any order. The $\backslash 0$ character cannot be specified as part of charset.

\section{Returns}

The wstrcreate_m function returns 0 if no runtime-constraints were violated. Otherwise, a nonzero value is returned.

\subsubsection{The const_strcreate_m function}

\section{Synopsis}

$$
\text { \#include <string_m.h> }
$$




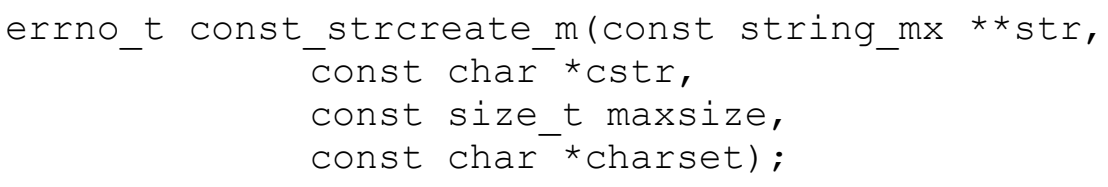

\section{Runtime-Constraints}

str shall not be a null pointer. charset shall not be an empty string (denoted by " "). Memory allocation for the string should succeed. Invalid characters are not present in the $\mathrm{C}$ string passed to the function.

\section{Description}

The const_strcreate $m$ function creates a constant managed string, given a conventional constant C string cstr (which may be null or empty). maxsize specifies the maximum size of the string in characters. If maxsize is 0 , the system-defined maximum length is used. charset restricts the set of allowable characters to those in the null-terminated byte string cstr (which may be empty). If charset is a null pointer, no restricted character set is defined. If specified, duplicated characters in a charset are ignored. Characters in the charset may be provided in any order. The $\backslash 0$ character cannot be specified as part of charset. The pointer to a constant string structure is returned to the caller by storing it in the parameter passed to the function.

This function acts as a wrapper function to the strcreate_m function. It passes all the arguments to the strcreate_m function to create a managed string. The pointer of that managed string is returned to the user as a pointer to the constant managed string.

\section{Returns}

The const_strcreate_m function returns 0 if no runtime-constraints were violated. Otherwise, a nonzero value is returned.

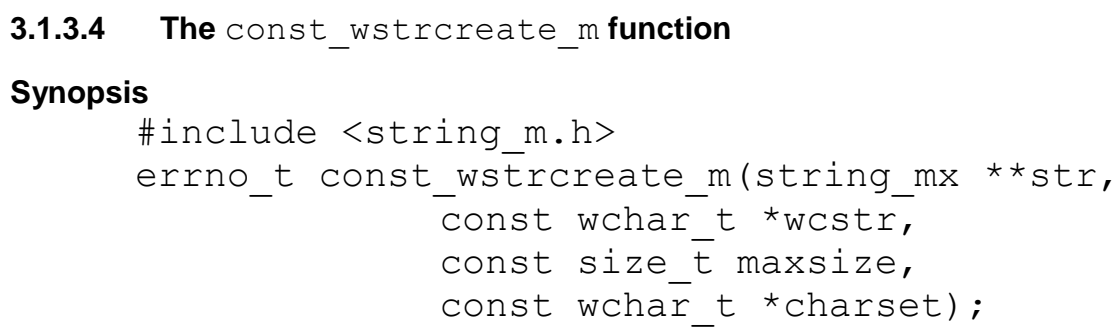

\section{Runtime-Constraints}

str shall not be a null pointer. charset shall not be an empty string (denoted by L""). Memory allocation for the string should succeed. Invalid characters are not present in the $\mathrm{C}$ string passed to the function.

\section{Description}

The const_wstrcreate_m function creates a constant managed string, referenced by str, given a wide character string wcstr (which may be null or empty). maxsize specifies the maximum size of the string in characters. If maxsize is zero, the system-defined maximum 
length is used. charset restricts the set of allowable characters to those in the wide character string wcstr (which may be empty). If charset is a null pointer, no restricted character set is defined. Characters in the charset may be provided in any order. The $\backslash 0$ character cannot be specified as part of charset.

\section{Returns}

The const_wstrcreate_m function returns 0 if no runtime-constraints were violated. Otherwise, a nonzero value is returned.

\subsubsection{The isntbs m Function}

\section{Synopsis}

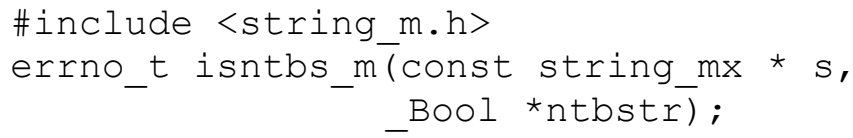

\section{Runtime-Constraints}

$s$ shall reference a valid managed string. ntbstr shall not be a null pointer.

\section{Description}

The isntbs $m$ function tests whether the managed string $\mathrm{s}$ is a null-terminated byte string and delivers this result in the parameter referenced by ntbstr, given the managed string s.

\section{Returns}

The isntbs_m function returns 0 if no runtime-constraints were violated. Otherwise, a nonzero value is returned.

\subsubsection{The iswide m Function \\ Synopsis

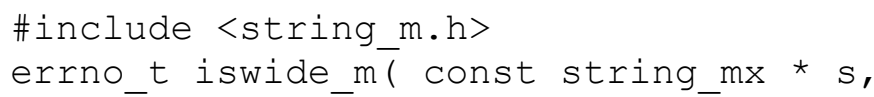

\section{Runtime-Constraints}

s shall reference a valid managed string. widestr shall not be a null pointer.

\section{Description}

The iswide_m function tests whether the managed string $\mathrm{s}$ is a wide character string and delivers this result in the parameter referenced by widestr, given the managed string $s$.

\section{Returns}

The iswide_m function returns 0 if no runtime-constraints were violated. Otherwise, a nonzero value is returned. 


\subsubsection{The strdelete m Function}

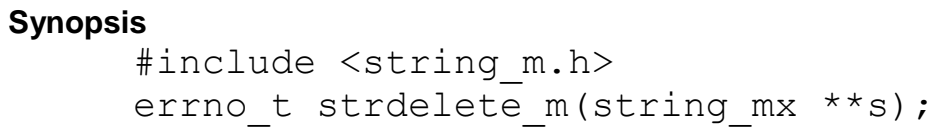

\section{Runtime-Constraints}

$\mathrm{s}$ shall not be a null pointer. ${ }^{\star}{ }^{\star} \mathrm{s}$ shall reference a valid managed string.

\section{Description}

The strdelete_m function deletes the managed string referenced by ${ }^{\star}{ }_{s}$ (which may be null or empty). $\mathrm{s}$ is set to a null pointer.

\section{Returns}

The strdelete_m function returns 0 if no runtime-constraints were violated. Otherwise, a nonzero value is returned.

\subsubsection{The strlen m Function}

\section{Synopsis}

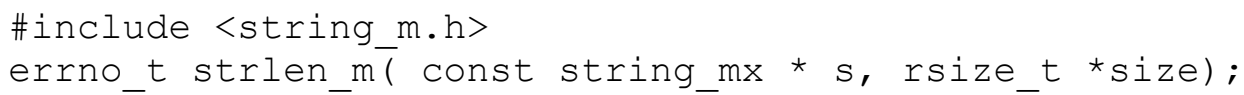

\section{Runtime-Constraints}

s shall reference a valid managed string. size shall not be a null pointer.

\section{Description}

The strlen_m function computes the length of the constant managed string $s$ and stores the result into the variable referenced by size. If the managed string is either null or empty, the length is computed as 0 . For a null-terminated byte string, the length is the number of bytes. For a wide character string, the length is the number of characters.

\section{Returns}

The strlen_m function returns 0 if no runtime-constraints were violated. Otherwise, a nonzero value is returned.

\subsubsection{Extracting a Conventional String}

\subsubsection{The cgetstr_m Function}

\section{Synopsis}

\#include <string_m.h>

errno_t cgetstr_m( const string_mx *s, const char **string);

\section{Runtime-Constraints}

s shall reference a valid managed string. string shall not be a null pointer. * string must be a null pointer. 


\section{Description}

The cgetstr_m function allocates storage for, and returns a pointer to, a null-terminated byte string represented by the managed string s and referenced by string. The caller is responsible for freeing * string when the null-terminated byte string is no longer required.

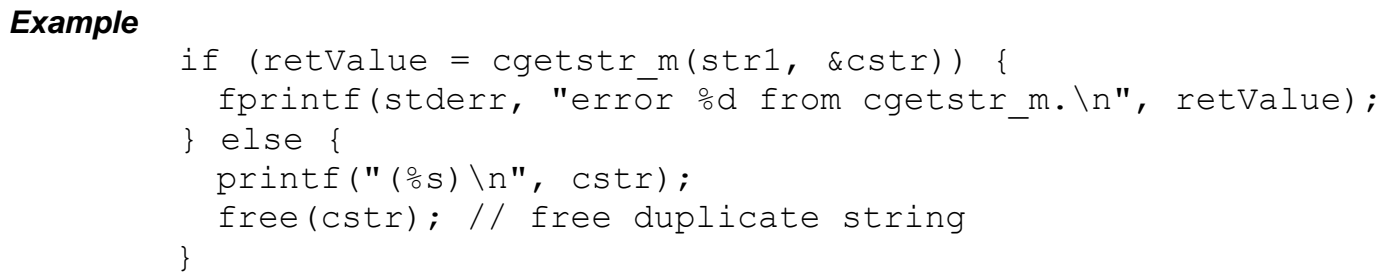

\section{Returns}

The cgetstr $m$ function returns 0 if no runtime-constraints were violated. Otherwise, a nonzero value is returned. If there is a runtime-constraint violation, ${ }^{*}$ string is set to a null pointer.

\subsubsection{The wgetstr_m Function}

\section{Synopsis}

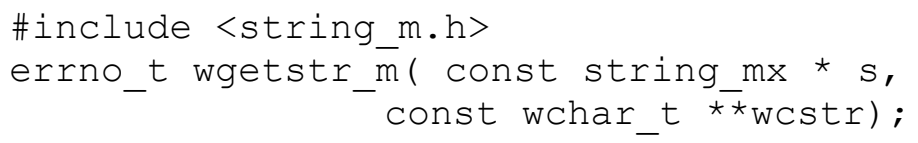

\section{Runtime-Constraints}

s shall reference a valid managed string. wCStr shall not be a null pointer. ${ }^{*}$ wCstr must be a null pointer.

\section{Description}

The wgetstr $m$ function delivers a wide character string into the variable referenced by wcstr, given the managed string $\mathrm{s}$. The caller is responsible for freeing * wCStr when the wide character string is no longer required.

\section{Returns}

The wgetstr_m function returns 0 if no runtime-constraints were violated. Otherwise, a nonzero value is returned. If there is a runtime-constraint violation, ${ }^{*}$ westr is set to a null pointer.

\subsubsection{The strdup_m Function}

\section{Synopsis}

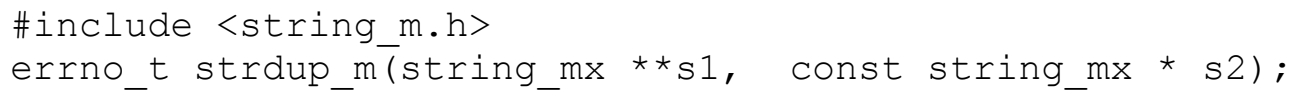

\section{Runtime-Constraints}

s1 shall not be a null pointer. s2 shall reference a valid managed string.

\section{Description}

The strdup_m function creates a duplicate of the managed string $s 2$ and stores it in $s 1$. The duplicate shall have the same set of valid characters and maximum length. 


\section{Returns}

The strdup_m function returns 0 if no runtime-constraints were violated. Otherwise, a nonzero value is returned.

\subsection{Copying Functions}

\subsubsection{Unbounded String Copy}

\subsubsection{The strcpy_m Function}

\section{Synopsis}

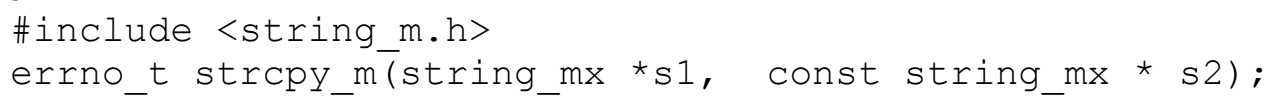

\section{Runtime-Constraints}

s1 and s2 shall reference valid managed strings.

\section{Description}

The strcpy_m function copies the managed string $s 2$ into the managed string $s 1$. Note that the set of valid characters and maximum length are not copied as these are attributes of $\mathrm{s} 1 .^{4}$

\section{Returns}

The strcpy_m function returns 0 if no runtime-constraints were violated. Otherwise, a nonzero value is returned.

\subsubsection{The cstrcpy_m Function}

\section{Synopsis}

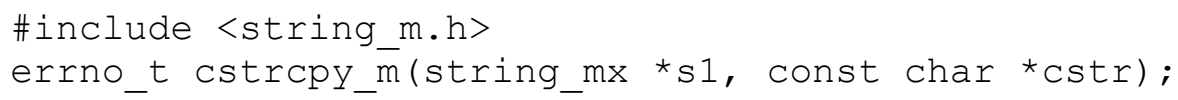

\section{Runtime-Constraints}

s1 shall reference a valid managed string.

\section{Description}

The cstrcpy_m function copies the string cstr into the managed string s 1 .

\section{Returns}

The cstrcpy_m function returns 0 if no runtime-constraints were violated. Otherwise, a nonzero value is returned.

\subsubsection{The wstrcpy m Function}

\section{Synopsis}

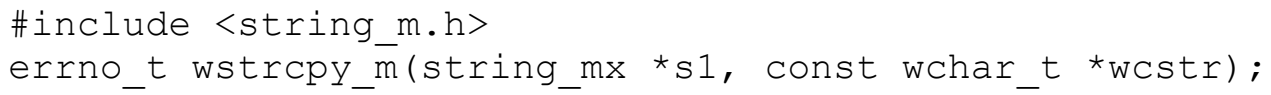

4 If $s 2$ contains characters that are not in the set of valid characters or exceeds the maximum length defined for s1, a runtime-constraint violation occurs as described in Section 2.6. 


\section{Runtime-Constraints}

s1 shall reference a valid managed string.

\section{Description}

The wstrcpy_m function copies the string wcstr into the managed string $s 1$.

\section{Returns}

The wstrcpy_m function returns 0 if no runtime-constraints were violated. Otherwise, a nonzero value is returned.

\subsubsection{The strncpy_m Function}

\section{Synopsis}

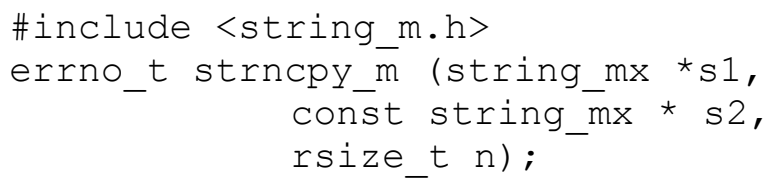

\section{Runtime-Constraints}

s1 and s2 shall reference valid managed strings.

\section{Description}

The strncpy_m function copies not more than $n$ characters from the managed string $s 2$ to the managed string $s 1$. If $s 2$ does not contain $n$ characters, the entire string is copied. If $s 2$ contains more than $n$ characters, $\mathrm{s} 1$ is set to the string containing the first $\mathrm{n}$ characters.

\section{Returns}

The strncpy_m function returns 0 if no runtime-constraints were violated. Otherwise, a nonzero value is returned.

\subsection{Concatenation Functions}

\subsubsection{Unbounded Concatenation}

\subsubsection{The strcat_m Function}

Synopsis

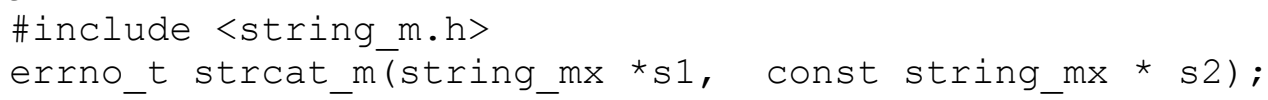

\section{Runtime-Constraints}

s1 and s2 shall reference valid managed strings.

\section{Description}

The strcat_m function concatenates the managed string $s 2$ onto the end of the managed string s1. 


\section{Returns}

The strcat_m function returns 0 if no runtime-constraints were violated. Otherwise, a nonzero value is returned.

\subsubsection{The cstrcat_m Function}

\section{Synopsis}

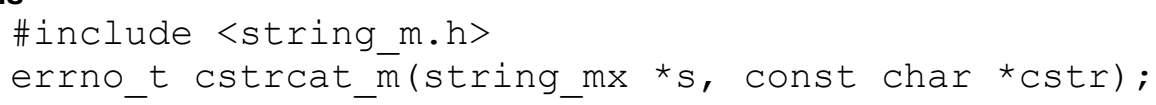

\section{Runtime-Constraints}

$\mathrm{s}$ shall reference a valid managed string.

\section{Description}

The cstrcat_m function concatenates the null-terminated byte string cstr onto the end of the managed string s. If cstr is a null pointer, this function returns without modifying s.

\section{Returns}

The cstrcat_m function returns 0 if no runtime-constraints were violated. Otherwise, a nonzero value is returned.

\subsubsection{The wstrcat_m Function}

\section{Synopsis}

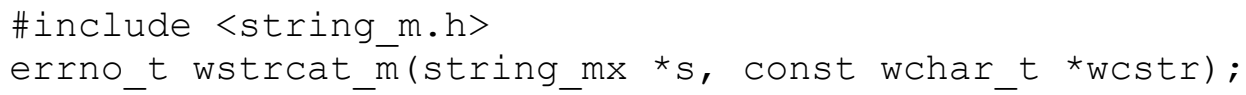

\section{Runtime-Constraints}

s shall reference a valid managed string. wCstr shall not be a null pointer.

\section{Description}

The wstrcat_m function concatenates the wide character string wcstr onto the end of the managed string s. If wCstr is a null pointer, this function returns without modifying s.

\section{Returns}

The wstrcat_m function returns 0 if no runtime-constraints were violated. Otherwise, a nonzero value is returned.

\subsubsection{Bounded Concatenation}

\subsubsection{The strncat_m Function}

\section{Synopsis}

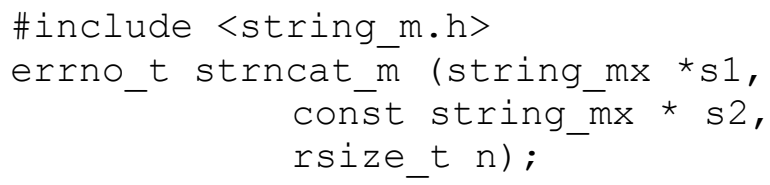




\section{Runtime-Constraints}

s1 and s2 shall reference valid managed strings.

\section{Description}

The strncat_m function appends not more than $n$ characters from the managed string $s 2$ to the end of the managed string $s 1$. If $s 2$ is a null pointer, strncat_m returns without modifying s1.

\section{Returns}

The strncat_m function returns 0 if no runtime-constraints were violated. Otherwise, a nonzero value is returned.

\subsubsection{The cstrncat_m Function}

\section{Synopsis}

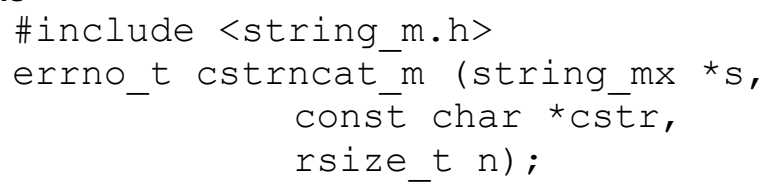

\section{Runtime-Constraints}

$\mathrm{s}$ shall reference a valid managed string.

\section{Description}

The cstrncat_m function appends not more than $n$ bytes from the null-terminated byte string cstr to the end of the managed string s. If cstr is null, cstrncat_m returns without modifying $s$. The cstrncat_m function guarantees that the resulting string $s$ is properly terminated.

\section{Returns}

The cstrncat_m function returns 0 if no runtime-constraints were violated. Otherwise, a nonzero value is returned.

\subsubsection{The wstrncat_m Function}

\section{Synopsis}

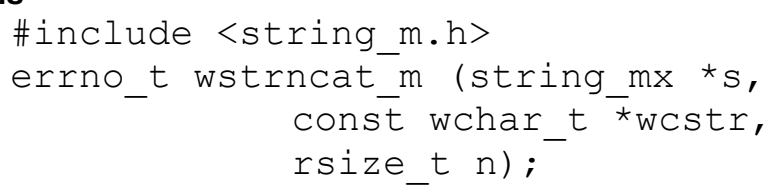

\section{Runtime-Constraints}

$s$ shall reference a valid managed string.

\section{Description}

The wstrncat_m function appends not more than $n$ characters from the wide character string wcstr to the end of the managed string s. If wcstr is a null pointer, the wstrncat_m func- 
tion returns without modifying $\mathrm{s}$. The wstrncat_m function guarantees that the resulting string $\mathrm{s}$ is properly terminated.

\section{Returns}

The wstrncat_m function returns 0 if no runtime-constraints were violated. Otherwise, a nonzero value is returned.

\subsection{Comparison Functions}

The sign of a nonzero value delivered by the comparison functions strcmp_m and strncmp_m is determined by the sign of the difference between the values of the first pair of characters (both interpreted as unsigned char but promoted to int) that differ in the objects being compared.

For the purpose of comparison, a null string is less than any other string, including an empty string. Null strings are equal, and empty strings are equal.

The set of valid characters defined for each string is not a factor in the evaluation; however, it is held as an invariant that each managed string contains only characters identified as valid for that string.

\subsubsection{Unbounded Comparison}

\subsubsection{The strcmp_m Function}

\section{Synopsis}

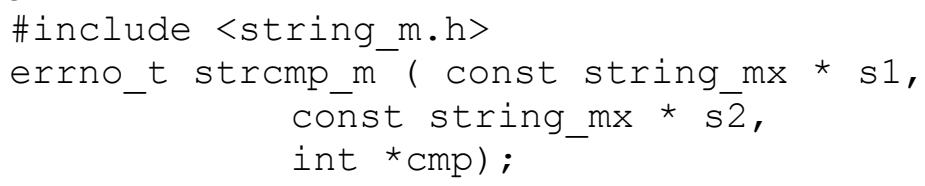

\section{Runtime-Constraints}

s1 and s2 shall reference valid managed strings. cmp shall not be a null pointer.

\section{Description}

The strcmp_m function compares the constant managed string $s 1$ to the constant managed string s2 and sets cmp to an integer value greater than, equal to, or less than 0 accordingly as s 1 is greater than, equal to, or less than $\mathrm{s} 2$.

\section{Returns}

The strcmp_m function returns 0 if no runtime-constraints were violated. Otherwise, a nonzero value is returned.

\subsubsection{The cstrcmp_m Function}

Synopsis

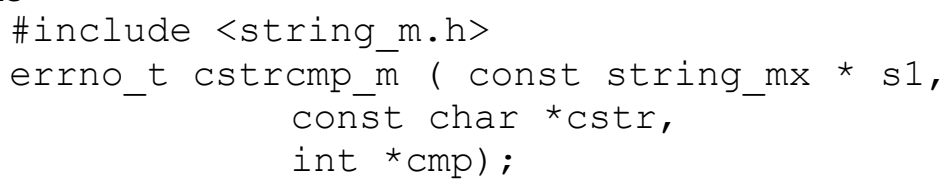




\section{Runtime-Constraints}

s1 shall reference a valid managed string. cmp shall not be a null pointer.

\section{Description}

The cstrcmp_m function compares the managed string $s 1$ to the null-terminated byte string cstr and sets $\mathrm{cmp}$ to an integer value greater than, equal to, or less than 0 accordingly as $\mathrm{s} 1$ is greater than, equal to, or less than cstr.

\section{Returns}

The cstrcmp_m function returns 0 if no runtime-constraints were violated. Otherwise, a nonzero value is returned.

\subsubsection{The wstrcmp_m Function}

\section{Synopsis}

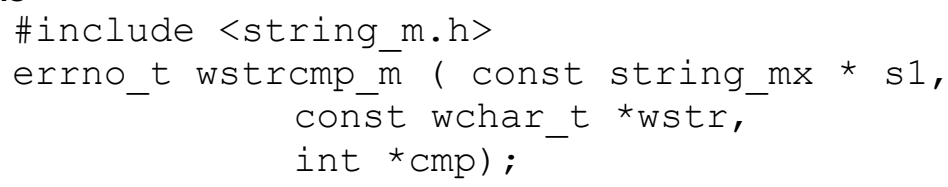

\section{Runtime-Constraints}

s1 shall reference a valid managed string. cmp shall not be a null pointer.

\section{Description}

The wstrcmp_m function compares the managed string $s 1$ to the wide character string wstr and sets cmp to an integer value greater than, equal to, or less than 0 accordingly as $s 1$ is greater than, equal to, or less than wstr.

\section{Returns}

The wstrcmp_m function returns 0 if no runtime-constraints were violated. Otherwise, a nonzero value is returned.

\subsubsection{Bounded String Comparison}

\subsubsection{The strncmp_m Function}

Synopsis

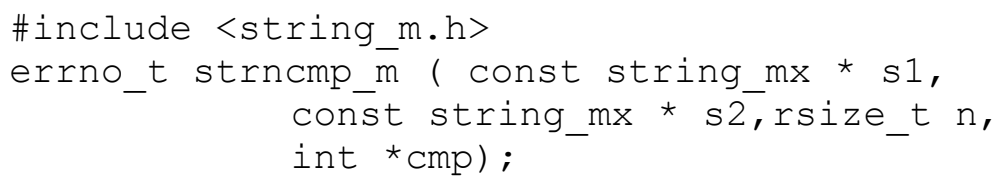

\section{Runtime-Constraints}

s1 and s2 shall reference valid managed strings. cmp shall not be a null pointer.

\section{Description}

The strncmp_m function compares not more than $n$ characters (characters that follow a null character are not compared) from the managed string $s 1$ to the managed string $s 2$ and sets $\mathrm{cmp}$ 
to an integer value greater than, equal to, or less than 0 accordingly as $s 1$ is greater than, equal to, or less than $s 2$. If $n$ is equal to 0 , the strncmp_m function sets $c m p$ to the integer value 0 , regardless of the contents of the string.

\section{Returns}

The strncmp_m function returns 0 if no runtime-constraints were violated. Otherwise, a nonzero value is returned.

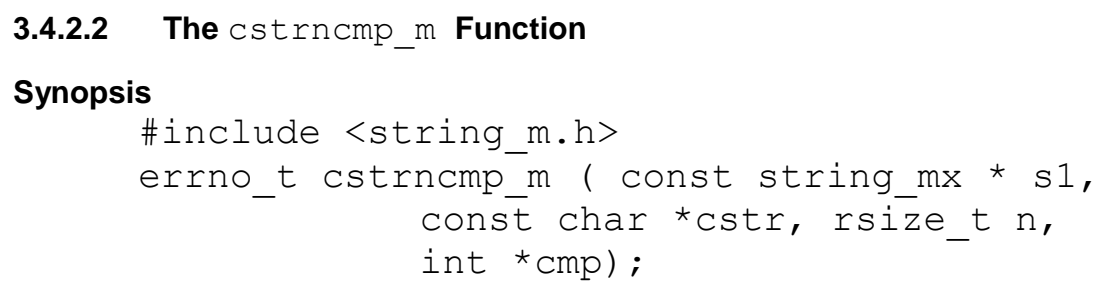

\section{Runtime-Constraints}

s1 shall reference a valid managed string. cmp shall not be a null pointer.

\section{Description}

The cstrncmp_m function compares not more than $n$ bytes (bytes that follow a null character are not compared) from the managed string $s 1$ to the null-terminated byte string cst $r$ and sets $\mathrm{cmp}$ to an integer value greater than, equal to, or less than 0 accordingly as $\mathrm{s} 1$ is greater than, equal to, or less than cstr. If $n$ is equal to 0 , the cstrncmp_m function sets $\mathrm{cmp}$ to the integer value 0 , regardless of the contents of the string.

\section{Returns}

The cstrncmp_m function returns 0 if no runtime-constraints were violated. Otherwise, a nonzero value is returned.

\subsubsection{The wstrncmp_m Function}

\section{Synopsis}

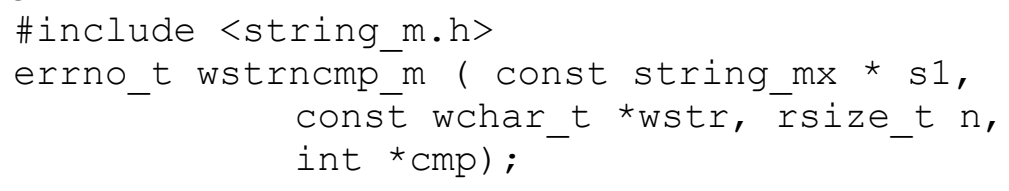

\section{Runtime-Constraints}

s1 shall reference a valid managed string. cmp shall not be a null pointer.

\section{Description}

The wstrncmp_m function compares not more than $n$ characters (characters that follow a null character are not compared) from managed string s 1 to the wide character string wstr and sets $\mathrm{cmp}$ to an integer value greater than, equal to, or less than 0 accordingly as $s 1$ is greater than, equal to, or less than wstr. If $n$ is equal to 0, the wstrncmp_m function sets cmp to the integer value 0 regardless of the contents of the string. 


\section{Returns}

The wstrncmp_m function returns 0 if no runtime-constraints were violated. Otherwise, a nonzero value is returned.

\subsection{Search Functions}

\subsubsection{The strtok_m Function}

\section{Synopsis}

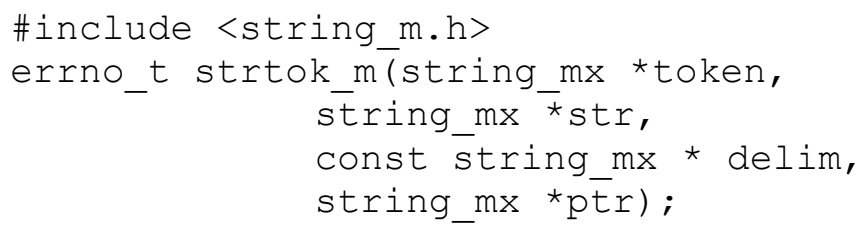

\section{Runtime-Constraints}

token, str, delim, and ptr shall reference valid managed strings.

\section{Description}

The strtok_m function scans the managed string str. The substring of str, up to but not including the first occurrence of any of the characters contained in the managed string delim, is returned as the managed string token. The remainder of the managed string str, after but not including the first character found from delim, is returned as the managed string ptr. If str does not contain any characters in delim (or if delim is either empty or null), token shall be set to str, and ptr will be set to the null string.

\section{Returns}

The strtok_m function returns 0 if there was no runtime-constraint violation. Otherwise, a nonzero value is returned.

\subsubsection{The cstrchr_m Function}

\section{Synopsis}

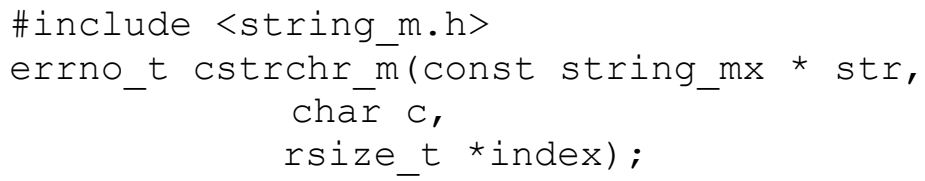

\section{Runtime-Constraints}

str shall reference valid managed strings.

\section{Description}

The cstrchr_m function scans the managed string str for the first occurrence of $c$. The parameter index is set to the first occurrence of character $\mathrm{c}$ in the string str. If $\mathrm{c}$ is not found in str, the index references to $\sim 0$. 


\section{Returns}

The cstrchr_m function returns 0 if there was no runtime-constraint violation. Otherwise, a nonzero value is returned.

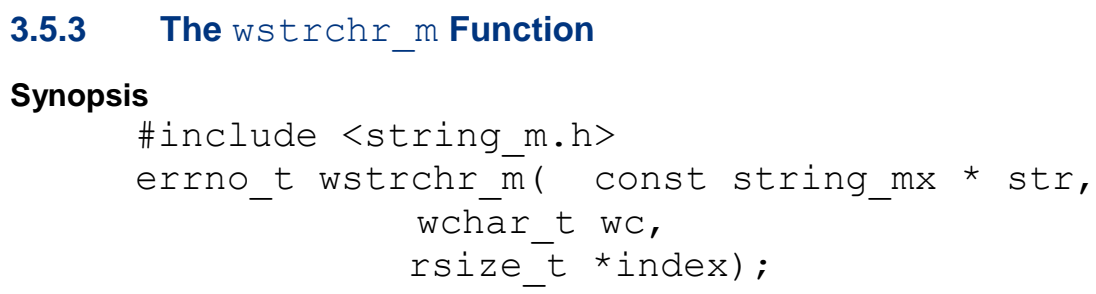

\section{Runtime-Constraints}

str shall reference valid managed strings.

\section{Description}

The wstrchr_m function scans the managed string str for the first occurrence of wc. The parameter index is set to the first occurrence of wide character $c$ in the string str. If $c$ is not found in str, the index references to $\sim 0$.

\section{Returns}

The wstrchr_m function returns 0 if there was no runtime-constraint violation. Otherwise, a nonzero value is returned.

\subsubsection{The strspn m Function}

\section{Synopsis}

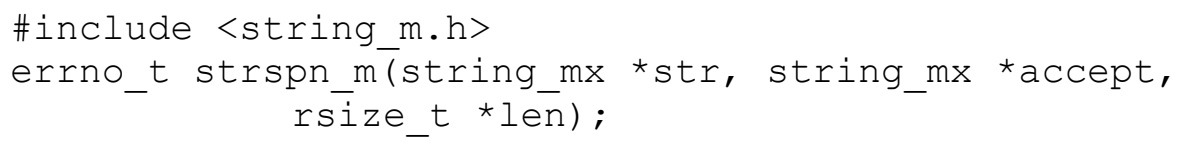

\section{Runtime-Constraints}

str and accept shall reference a valid managed string. len shall not be a null pointer.

\section{Description}

The strspn_m function computes the length of the maximum initial segment of the managed string str, which consists entirely of characters from the managed string accept. The strspn_m function sets * len to this length. If the managed string str is null or empty, * len is set to 0 .

\section{Returns}

The strspn_m function returns 0 if there was no runtime-constraint violation. Otherwise, a nonzero value is returned.

\subsubsection{The cstrspn m Function}

\section{Synopsis}

$$
\text { \#include <string_m.h> }
$$




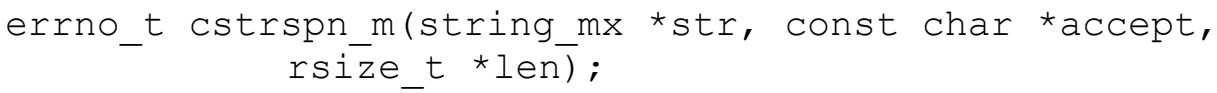

\section{Runtime-Constraints}

str and accept shall reference a valid managed string. len shall not be a null pointer.

\section{Description}

The cstrspn_m function computes the length of the maximum initial segment of the managed string str, which consists entirely of characters from the string accept. The cstrspn_m function sets ${ }^{*}$ len to this length. If the managed string str is null or empty, ${ }^{*}$ len is set to 0 .

\section{Returns}

The cstrspn_m function returns 0 if there was no runtime-constraint violation. Otherwise, a nonzero value is returned.

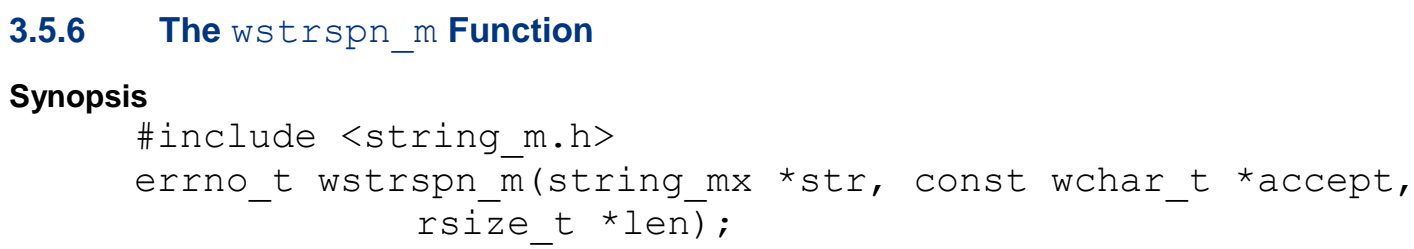

\section{Runtime-Constraints}

str and accept shall reference a valid managed string. len shall not be a null pointer.

\section{Description}

The wstrspn $m$ function computes the length of the maximum initial segment of the managed string str, which consists entirely of characters from the string accept. The wstrspn_m function sets * len to this length. If the managed string str is null or empty, * len is set to 0 .

\section{Returns}

The wstrspn_m function returns 0 if there was no runtime-constraint violation. Otherwise, a nonzero value is returned.

\subsubsection{The strcspn m Function}

\section{Synopsis}

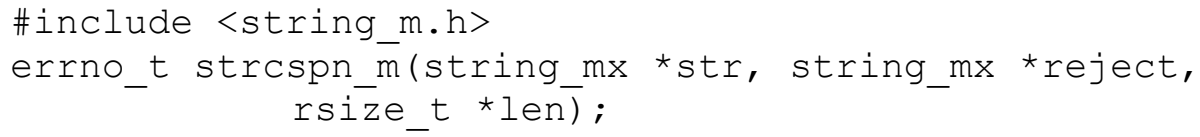

\section{Runtime-Constraints}

str and reject shall reference valid managed strings. Ien shall not be a null pointer.

\section{Description}

The strcspn_m function computes the length of the maximum initial segment of the managed string str, which consists entirely of characters not from the managed string reject. The strcspn_m function sets *len to this length. If the managed string str is null or empty, 
* Ien is set to 0 . If the managed string reject is null or empty, ${ }^{*}$ len is set to the length of str.

\section{Returns}

The strcspn_m function returns 0 if there was no runtime-constraint violation. Otherwise, a nonzero value is returned.

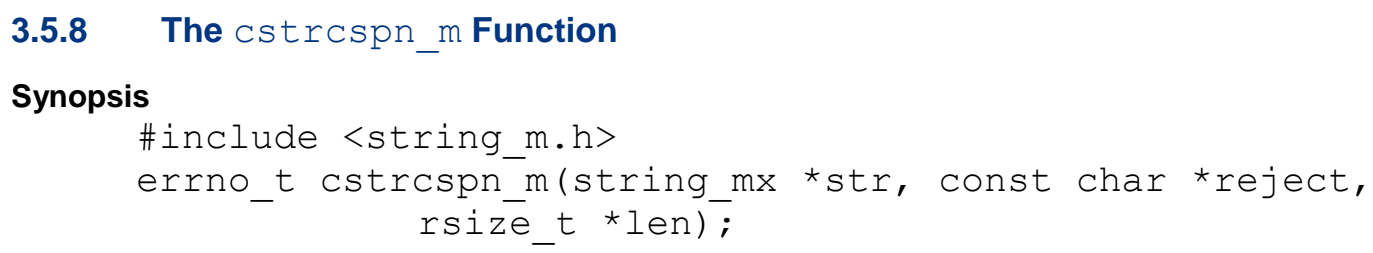

Runtime-Constraints

str and reject shall reference valid managed strings. len shall not be a null pointer.

\section{Description}

The cstrcspn_m function computes the length of the maximum initial segment of the managed string str, which consists entirely of characters not from the managed string reject. The cstrcspn_m function sets *len to this length. If the managed string str is null or empty, ${ }^{\star} l e n$ is set to 0 . If the managed string reject is null or empty, *len is set to the length of str.

\section{Returns}

The cstrcspn_m function returns 0 if there was no runtime-constraint violation. Otherwise, a nonzero value is returned.

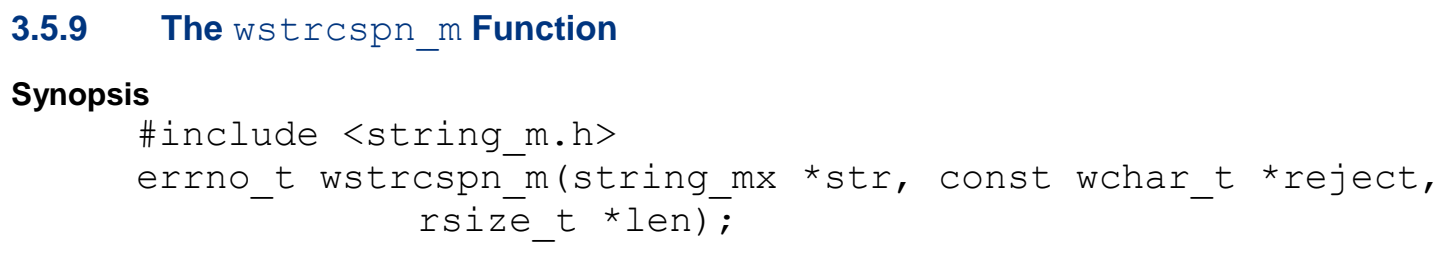

Runtime-Constraints

str and reject shall reference valid managed strings. len shall not be a null pointer.

\section{Description}

The wstrcspn_m function computes the length of the maximum initial segment of the managed string str, which consists entirely of characters not from the managed string reject. The wstrcspn_m function sets * len to this length. If the managed string str is null or empty, *len is set to 0 . If the managed string reject is null or empty, *len is set to the length of str. 


\section{Returns}

The wstrcspn_m function returns 0 if there was no runtime-constraint violation. Otherwise, a nonzero value is returned.

\subsection{Configuration Functions}

\subsubsection{The setcharset_m Function}

\section{Synopsis}

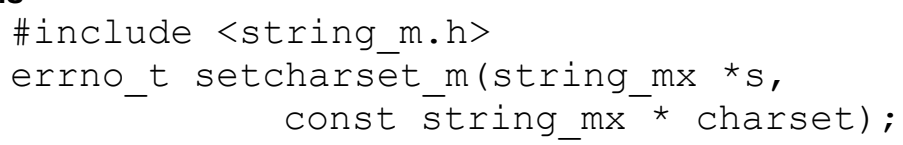

\section{Runtime-Constraints}

$\mathrm{s}$ shall reference a valid managed string.

\section{Description}

The setcharset_m function sets the subset of allowable characters to those in the managed string charset, which may be null or empty. If charset is a null pointer or the managed string represented by charset is null, a restricted subset of valid characters is not enforced. If the managed string charset is empty, then only empty or null strings can be created.

\section{Returns}

The setcharset_m function returns 0 if no runtime-constraints were violated. Otherwise, a nonzero value is returned.

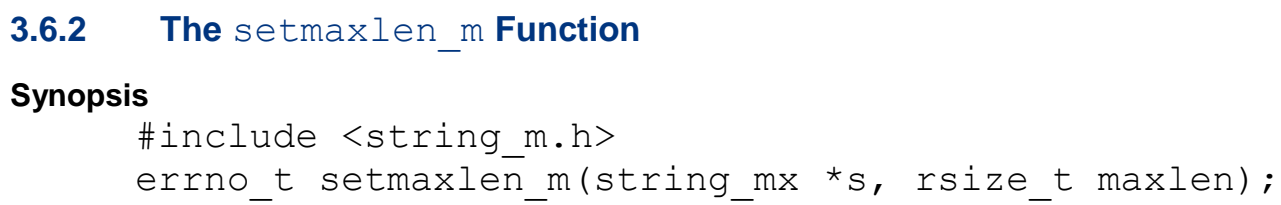

\section{Runtime-Constraints}

$\mathrm{s}$ shall reference a valid managed string.

\section{Description}

The setmaxlen_m function sets the maximum length of the managed string $\mathrm{s}$. If maxlen is 0 , the system-defined maximum length is used.

\section{Returns}

The setmaxlen_m function returns 0 if no runtime-constraints were violated. Otherwise, a nonzero value is returned.

\subsection{Functions Derived from printf}

These functions are the managed string equivalents to the printf-derived functions in $\mathrm{C}$. 


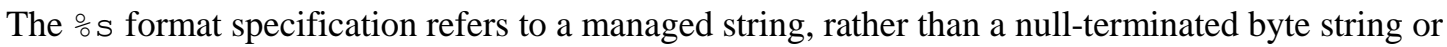
wide character string. The format specification $\frac{1}{2} \mathrm{~s}$ indicates that the managed string should be output as a wide character string. The format specification $\circ \mathrm{hs}$ indicates that the managed string should be output as a null-terminated byte string. All printf-derived functions will output a null-terminated byte string if (1) any specified output stream is byte oriented and (2) the format string and all argument strings are null-terminated byte strings; otherwise the output will be a wide character string.

Applying a byte output function to a wide-oriented stream or applying a wide character output function to a byte-oriented stream will result in a runtime-constraint error.

The $\frac{\circ}{\mathrm{n}}$ format specification is not recognized.

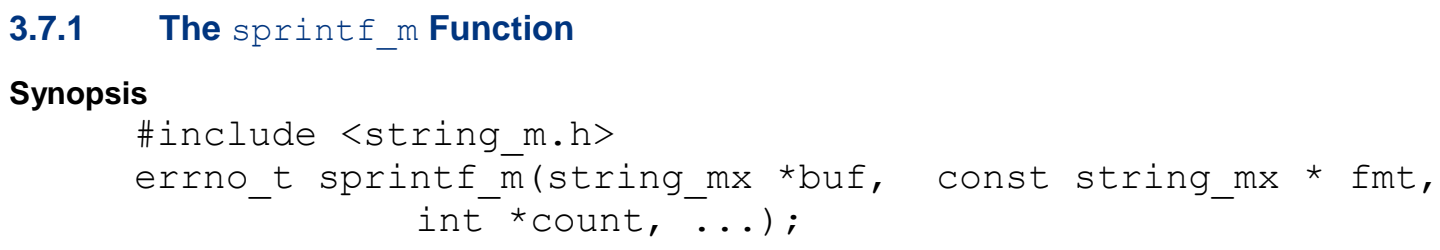

\section{Runtime-Constraints}

buf and fmt shall reference valid managed strings. The managed string fmt shall be a valid format compatible with the arguments after fmt.

\section{Description}

The sprintf_m function formats its parameters after the third parameter into a string according to the format contained in the managed string fmt and stores the result in the managed string buf.

If not a null pointer, ${ }^{\star}$ count is set to the number of characters written in buf, not including the terminating null character.

\section{Returns}

The sprintf_m function returns 0 if no runtime-constraints were violated. Otherwise, a nonzero value is returned.

\subsubsection{The vsprintf_m Function}

\section{Synopsis}

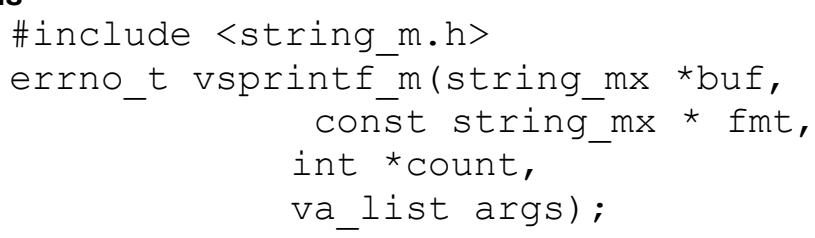

\section{Runtime-Constraints}

buf and fmt shall reference a valid managed string. fmt shall not be a null pointer. The managed string fmt shall be a valid format compatible with the arguments args. 


\section{Description}

The vsprintf_m function formats its parameters args into a string according to the format contained in the managed string fmt and stores the result in the managed string buf.

If not a null pointer, ${ }^{\star}$ count is set to the number of characters written in buf, not including the terminating null character.

\section{Returns}

The vsprintf_m function returns 0 if no runtime-constraints were violated. Otherwise, a nonzero value is returned.

\subsubsection{The printf_m Function}

\section{Synopsis}

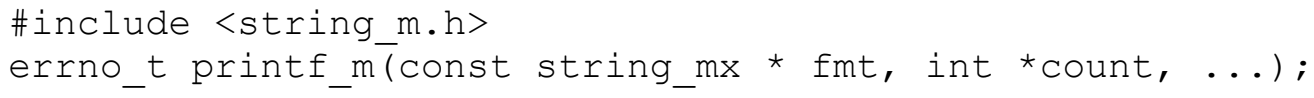

\section{Runtime-Constraints}

fmt shall reference a valid managed string. fmt shall not be a null pointer. The managed string fmt shall be a valid format compatible with the arguments after fmt.

\section{Description}

The printf_m function formats its parameters after the second parameter into a string according to the format contained in the managed string fmt and outputs the result to standard output.

If not a null pointer, ${ }^{*}$ count is set to the number of characters transmitted.

\section{Returns}

The printf_m function returns 0 if no runtime-constraints were violated. Otherwise, a nonzero value is returned.

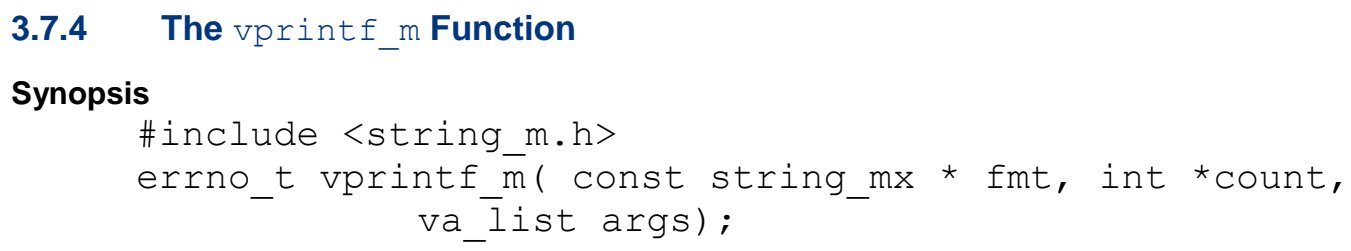

\section{Runtime-Constraints}

fmt shall reference a valid managed string. fmt shall not be a null pointer. The managed string fmt shall be a valid format compatible with the arguments args.

\section{Description}

The vprintf_m function formats its parameters args into a string according to the format contained in the managed string fmt and outputs the result to standard output.

If not a null pointer, ${ }^{\star}$ count is set to the number of characters transmitted. 


\section{Returns}

The vprintf $m$ function returns 0 if no runtime-constraints were violated. Otherwise, a nonzero value is returned.

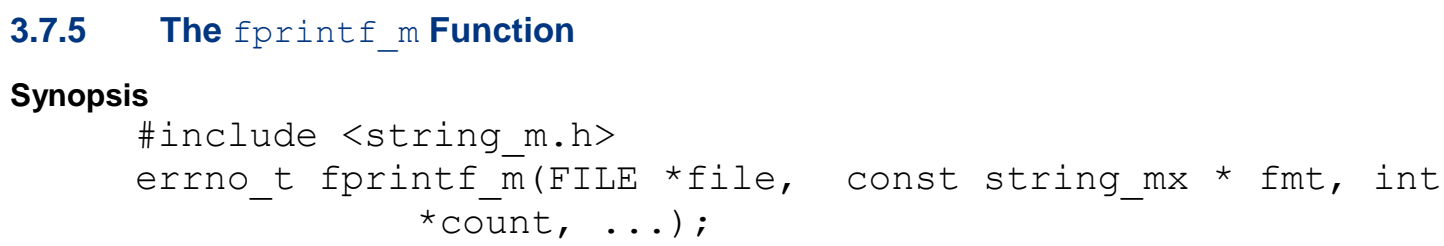

\section{Runtime-Constraints}

fmt shall reference a valid managed string. fmt shall not be a null pointer. The managed string fmt shall be a valid format compatible with the arguments after fmt. file shall not be a null pointer.

If not a null pointer, ${ }^{\star}$ count is set to the number of characters transmitted.

\section{Description}

The fprintf_m function formats its parameters after the third parameter into a string according to the format contained in the managed string fmt and outputs the result to file.

\section{Returns}

The fprintf_m function returns 0 if no runtime-constraints were violated. Otherwise, a nonzero value is returned.

\subsubsection{The vfprintf_m Function}

\section{Synopsis}

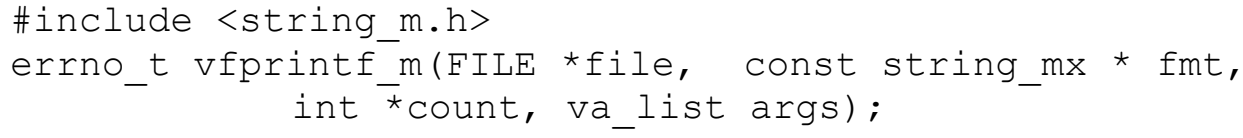

\section{Runtime-Constraints}

fmt shall reference a valid managed string. fmt shall not be a null pointer. The managed string fmt shall be a valid format compatible with the arguments args. file shall not be a null pointer.

\section{Description}

The vfprintf_m function formats its parameters args into a string according to the format contained in the managed string fmt and outputs the result to file.

If not a null pointer, ${ }^{\star}$ count is set to the number of characters transmitted.

\section{Returns}

The vfprintf_m function returns 0 if no runtime-constraints were violated. Otherwise, a nonzero value is returned. 


\subsection{Functions Derived from scanf}

These functions are the managed string equivalents to the scanf-derived functions in C. Managed string format strings differ from standard $\mathrm{C}$ format strings primarily in that they are represented as managed strings. The $\frac{\circ}{S}$ specification refers to a managed string rather than a nullterminated byte string or wide character string. The use of char* or wchar_t* pointers in the varargs list will result in a runtime-constraint if detected. The managed string read by $\circ s$ is created as a null-terminated byte string if the input string is a null-terminated byte string or the input stream has byte orientation; otherwise a wide character string is created. The format specification $\frac{\circ}{2} \mathrm{~s}$ indicates that the managed string should be created as a wide character string. The format specification $\circ \mathrm{hs}$ indicates that the managed string should be created as a null-terminated byte string.

Applying a byte input function to a wide-oriented stream or applying a wide character input function to a byte-oriented stream will result in a runtime-constraint error.

\subsubsection{The sscanf_m Function}

\section{Synopsis}

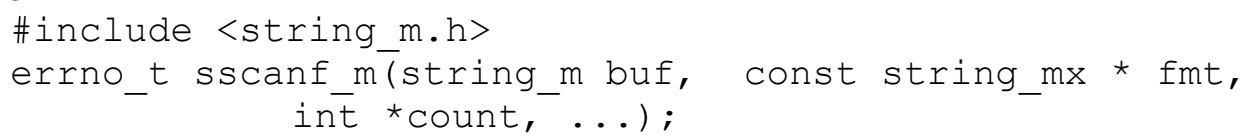

\section{Runtime-Constraints}

buf and fmt shall reference a valid managed string. fmt shall not be a null pointer. The managed string fmt shall be a valid format compatible with the arguments after fmt.

\section{Description}

The sscanf_m function processes the managed string buf according to the format contained in the managed string fmt and stores the results using the arguments after count.

If not a null pointer, ${ }^{*}$ count is set to the number of input items assigned, which can be fewer than provided for, or even zero, in the event of an early matching failure.

\section{Returns}

The sscanf_m function returns 0 if no runtime-constraints were violated. Otherwise, a nonzero value is returned.

\subsubsection{The vsscanf $m$ Function}

\section{Synopsis}

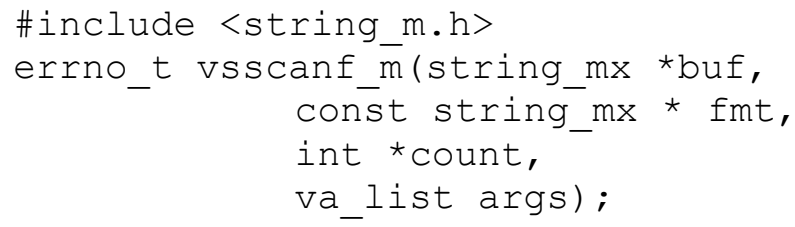




\section{Runtime-Constraints}

buf and fmt shall reference a valid managed string. fmt shall not be a null pointer. The managed string fmt shall be a valid format compatible with the arguments args.

\section{Description}

The vsscanf $m$ function processes the managed string buf according to the format contained in the managed string fmt and stores the results using the arguments in args.

If not a null pointer, ${ }^{*}$ count is set to the number of input items assigned, which can be fewer than provided for, or even zero, in the event of an early matching failure.

\section{Returns}

The vsscanf_m function returns 0 if no runtime-constraints were violated. Otherwise, a nonzero value is returned.

\subsubsection{The scanf_m Function}

\section{Synopsis}

\#include <string_m.h>

errno_t scanf_m( const string_mx * fmt, int *count, ...);

\section{Runtime-Constraints}

fmt shall reference a valid managed string. fmt shall not be a null pointer. The managed string fmt shall be a valid format compatible with the arguments after count.

\section{Description}

The scanf_m function processes input from standard input according to the format contained in the managed string fmt and stores the results using the arguments after count.

If not null, * count is set to the number of input items assigned, which can be fewer than provided for, or even zero, in the event of an early matching failure.

\section{Returns}

The scanf_m function returns 0 if no runtime-constraints were violated. Otherwise, a nonzero value is returned.

\subsubsection{The vscanf_m Function}

Synopsis

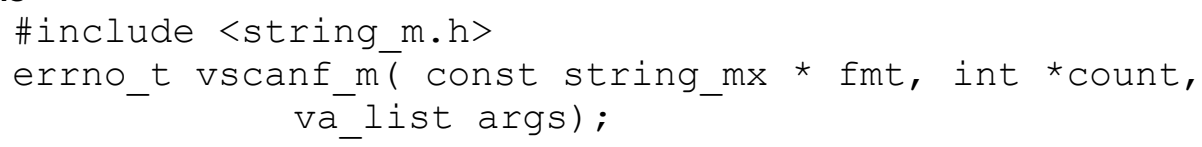

\section{Runtime-Constraints}

fmt shall reference a valid managed string. fmt shall not be a null pointer. The managed string fmt shall be a valid format compatible with the arguments args. 


\section{Description}

The vscanf_m function processes input from standard input according to the format contained in the managed string fmt and stores the results using the arguments in args.

If not null, ${ }^{*}$ count is set to the number of input items assigned, which can be fewer than provided for, or even zero, in the event of an early matching failure.

\section{Returns}

The vscanf_m function returns 0 if no runtime-constraints were violated. Otherwise, a nonzero value is returned.

\subsubsection{The fscanf_m Function}

\section{Synopsis}

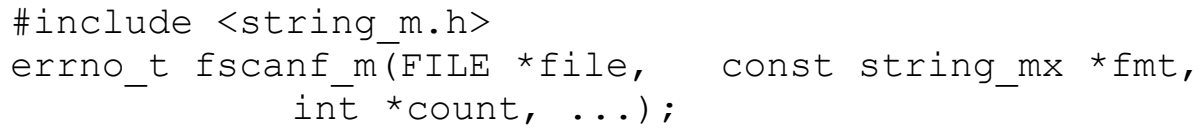

\section{Runtime-Constraints}

fmt shall reference a valid managed string. fmt shall not be a null pointer. The managed string fmt shall be a valid format compatible with the arguments after count. file shall not be a null pointer.

\section{Description}

The $f$ scanf_m function processes input from $f i l e$ according to the format contained in the managed string fmt and stores the results using the arguments after count.

If not a null pointer, ${ }^{*}$ count is set to the number of input items assigned, which can be fewer than provided for, or even zero, in the event of an early matching failure.

\section{Returns}

The $f$ scanf_m function returns 0 if no runtime-constraints were violated. Otherwise, a nonzero value is returned.

\subsubsection{The vfscanf $m$ Function}

\section{Synopsis}

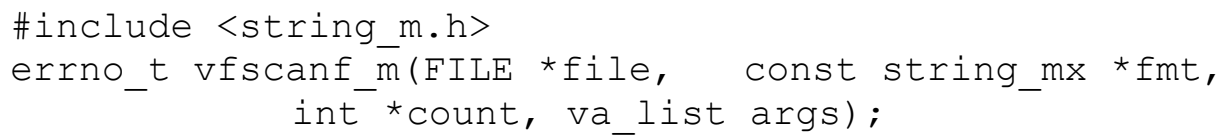

\section{Runtime-Constraints}

fmt shall reference a valid managed string. fmt shall not be a null pointer. The managed string fmt shall be a valid format compatible with the arguments after count. file shall not be a null pointer. 


\section{Description}

The vfscanf_m function processes input from $f i l e$ according to the format contained in the managed string fmt and stores the results using the arguments after count.

If not a null pointer, ${ }^{*}$ count is set to the number of input items assigned, which can be fewer than provided for, or even zero, in the event of an early matching failure.

\section{Returns}

The vfscanf_m function returns 0 if no runtime-constraints were violated. Otherwise, a nonzero value is returned.

\subsection{String Slices}

\subsubsection{The strslice_m Function}

\section{Synopsis}

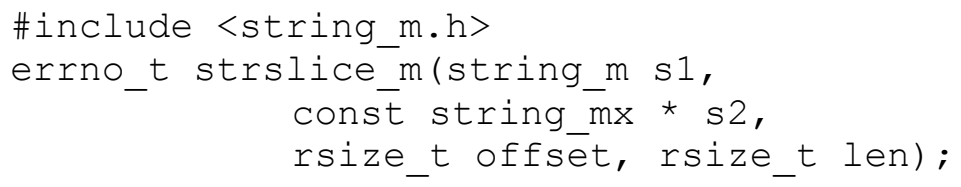

\section{Runtime-Constraints}

s1 and s2 shall reference valid managed strings. There shall be sufficient memory to store the result.

\section{Description}

The strslice_m function takes up to len characters from s2, starting at the off set character in the string, and stores the result in $\mathrm{s} 1$. If there are insufficient characters to copy len characters, all available characters are copied. If off set is greater than the number of characters in $\mathrm{s} 2, \mathrm{~s} 1$ is set to the null string. If off set is equal to the number of characters in s2 or len is 0 , $\mathrm{s} 1$ is set to the empty string.

\section{Returns}

The strslice_m function returns 0 if no runtime-constraints were violated. Otherwise, a nonzero value is returned.

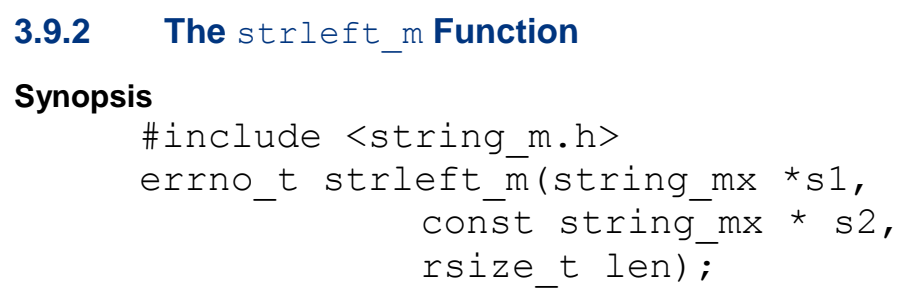

Runtime-Constraints

s1 and s2 shall reference valid managed strings. There shall be sufficient memory to store the result. 


\section{Description}

The strleft_m function copies up to len characters from the start of the managed string $\mathrm{s} 2$ to the managed string $s 1$. If $s 2$ does not have len characters, the entire string is copied. If $s 2$ is a null string, $s 1$ is set to the null string.

\section{Returns}

The strleft_m function returns 0 if no runtime-constraints were violated. Otherwise, a nonzero value is returned.

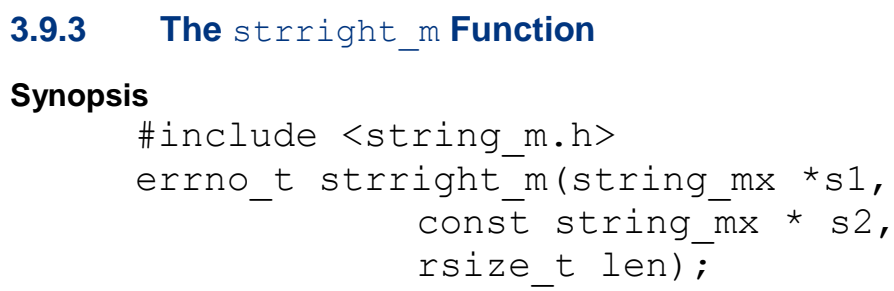

\section{Runtime-Constraints}

s1 and s2 shall reference valid managed strings. There shall be sufficient memory to store the result.

\section{Description}

The strright_m function copies up to the last len characters from the managed string $\mathrm{s} 2$ to the managed string $s 1$. If $s 2$ does not have len characters, the entire string is copied. If $s 2$ is a null string, $s 1$ is set to the null string.

\section{Returns}

The strright_m function returns 0 if no runtime-constraints were violated. Otherwise, a nonzero value is returned.

\subsubsection{The cchar_m Function}

\section{Synopsis}

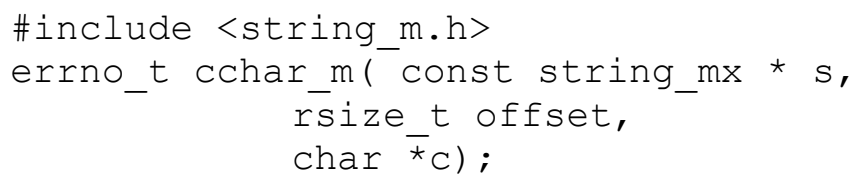

\section{Runtime-Constraints}

s shall reference a valid managed string. $c$ shall not be a null pointer. of f set shall be less than the length of the managed string $\mathrm{s}$. The character to be returned in $\mathrm{c}$ shall be representable as a char.

\section{Description}

The cchar_m function sets $c$ to the off set character (the first character having an off set of 0 ) in the managed string $s$. 


\section{Returns}

The cchar_m function returns 0 if no runtime-constraints were violated. Otherwise, a nonzero value is returned.

\subsubsection{The wchar_m Function}

Synopsis

\#include <string_m.h>

errno_t wchar_m( const string_mx * $s$,

rsize $t$ offset,

wchar_t $\left.{ }^{*} \mathrm{wC}\right)$;

\section{Runtime-Constraints}

s1 shall reference a valid managed string. wC shall not be a null pointer. offset shall be less than the length of the managed string $\mathrm{s} 1$.

\section{Description}

The wchar_m function sets wc to the offset character (the first character having an off set of 0 ) in the managed string $s$.

\section{Returns}

The wchar_m function returns 0 if no runtime-constraints were violated. Otherwise, a nonzero value is returned. 
36 | CMU/SEI-2010-TR-018 


\section{References}

URLs are valid as of the publication date of this document.

\section{[CERT 2009]}

CERT. Managed String Library. http://www.cert.org/secure-coding/managedstring.html (2009).

\section{[ISO/IEC 1999]}

International Organization for Standardization, International Electrotechnical Commission. ISO/IEC 9899:1999, Programming Languages - C. http://www.open-std.org/JTC1/SC22/WG14 /www/docs/n1124.pdf (May 6, 2005). 
38 | CMU/SEI-2010-TR-018 


\section{REPORT DOCUMENTATION PAGE \\ Form Approved

Public reporting burden for this collection of information is estimated to average 1 hour per response, including the time for reviewing instructions, searching existing data sources, gathering and maintaining the data needed, and completing and reviewing the collection of information. Send comments regarding this burden estimate or any other aspect of this collection of information, including suggestions for reducing this burden, to Washington Headquarters Services, Directorate for information Operations and Reports, 1215 Jefferson Davis Highway, Suite 1204, Arlington, VA 22202-4302, and to the Office of Management and Budget, Paperwork Reduction Project (0704-0188), Washington, DC 20503.

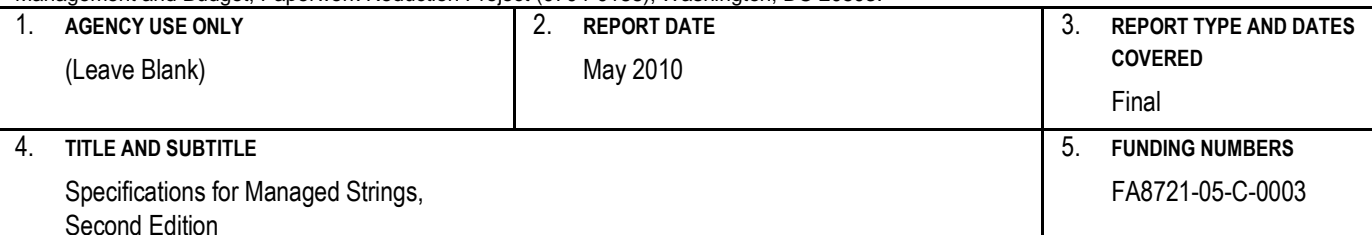

6. AUTHOR(S)

Hal Burch, Fred Long, Raunak Rungta, Robert Seacord, David Svoboda

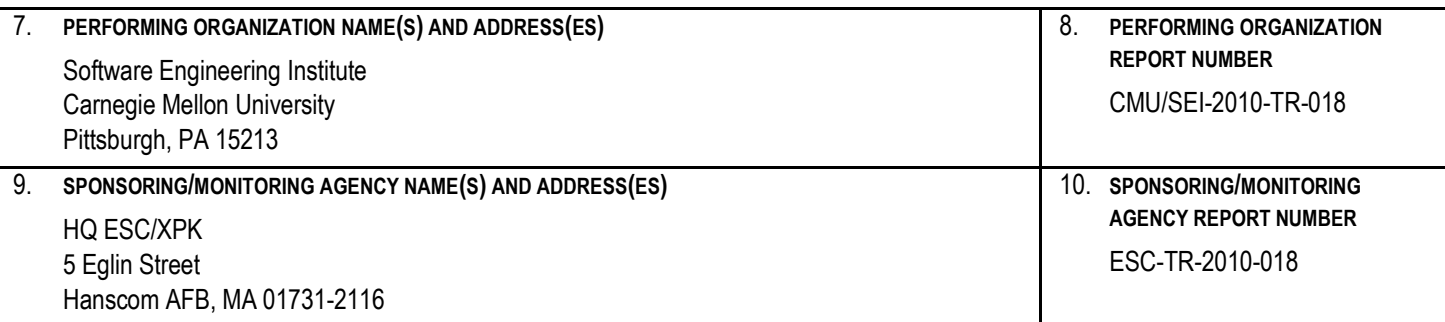

11. SUPPLEMENTARY NOTES

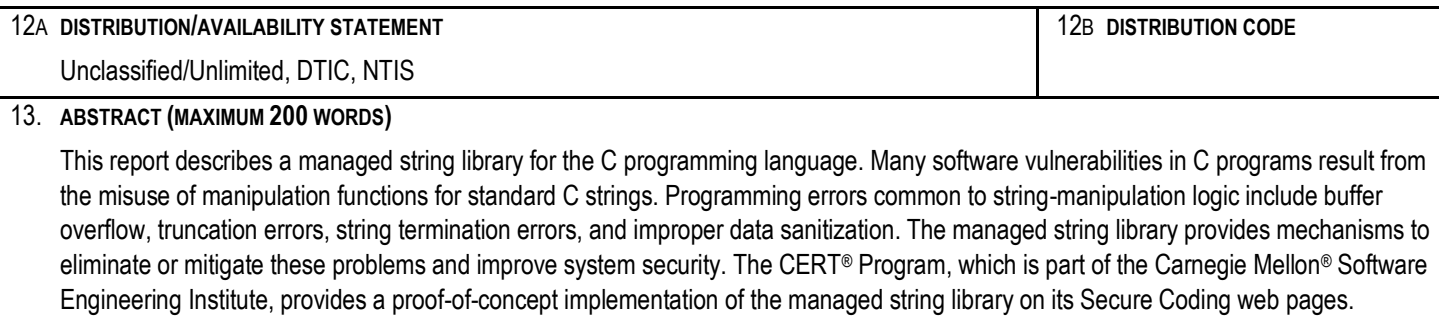

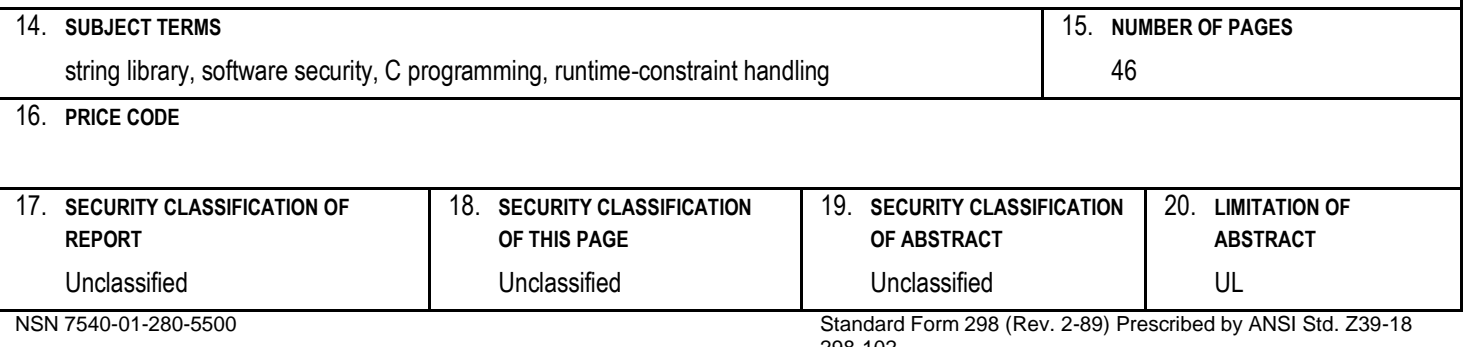

NSN 7540-01-280-5500 